\title{
Effects of fentanyl on pain and motor behaviors following a collagenase-induced intracerebral hemorrhage in rats
}

This article was published in the following Dove Press journal:

Journal of Pain Research

15 November 2016

Number of times this article has been viewed

\author{
Laurence Saine' \\ Pierre Hélie ${ }^{2}$ \\ Pascal Vachon' \\ 'Department of Veterinary \\ Biomedicine, Faculty of Veterinary \\ Medicine, ${ }^{2}$ Department of Pathology \\ and Microbiology, Faculty of \\ Veterinary Medicine, University of \\ Montreal, Saint-Hyacinthe, Quebec, \\ Canada
}

Purpose: Intracerebral hemorrhage $(\mathrm{IH})$ and cephalalgia are common consequences of traumatic brain injury. One of the primary obstacles for patient recovery is the paucity of treatments to support an appropriate analgesic protocol. The present study aimed to assess pain and motor behaviors following different doses of fentanyl on a rat model of IH.

Methods: Twenty-one male Sprague Dawley rats underwent a stereotaxic surgery to produce a collagenase-induced $\mathrm{IH}$ in the right caudoputamen nucleus. The control group $(n=6)$ received saline subcutaneously (SC), and experimental groups received either $5(n=6), 10(n=6)$, or 20 $(\mathrm{n}=3) \mu \mathrm{g} / \mathrm{kg}$ of fentanyl SC, 2 hours following surgery and on 2 subsequent days. Only 3 animals received $20 \mu \mathrm{g} / \mathrm{kg}$ because this dose caused catalepsy for 15-20 minutes following the injection. The rat grimace scale, a neurological examination, balance beam test, and rotarod test were performed for 5 consecutive days postoperatively to evaluate pain and motor performance. At the end of the experimentation, the brains were evaluated to determine hematoma volume, and the number of reactive astrocytes and necrotic neurons.

Results: When compared to controls, the grimace scale showed that $5 \mu \mathrm{g} / \mathrm{kg}$ fentanyl significantly alleviated pain on day 2 only $(P<0.01)$ and that $10 \mu \mathrm{g} / \mathrm{kg}$ alleviated pain on days $1(P<0.01)$, $2(P<0.001)$, and $3(P<0.01)$. For the rotarod test, only the $10 \mu \mathrm{g} / \mathrm{kg}$ group showed significant decreases in performance on days $5(P<0.05)$ and $6(P<0.02)$. The neurological examination was not significantly different between the groups, but only the hopping test showed poor recuperation for the 5 and $10 \mu \mathrm{g} / \mathrm{kg}$ fentanyl group when compared to saline $(P<0.01)$. No differences were found between the groups for the balance beam test, the histopathological results.

Conclusion: Fentanyl, at a dose of $10 \mu \mathrm{g} / \mathrm{kg} \mathrm{SC}$, provides substantial analgesia following a collagenase-induced IH in rats; however, it can alter motor performance following analgesic treatments.

Keywords: analgesia, brain trauma, cephalagia, grimace scale, histopathology, neurological exam

\section{Introduction}

Traumatic brain injury (TBI) is one of the major causes of invalidity and death in the society. Neurologic dysfunctions following brain injuries are often minimal immediately following the impact but a progressive deterioration occurs with time. ${ }^{1,2}$ Intracerebral hemorrhage (IH) is the most common and predominant consequence of TBI. The primary obstacle for patient recovery is the paucity of efficient treatments and the lack of publications to support treatment option. ${ }^{3,4}$ Traumatic IH is a multifaceted condition which has a complex physiopathology. Mechanisms involved include neuroinflammation, breakdown of the brain-blood barrier (BBB), and an increased intracranial pressure. One nonnegligible aspect is the pain associated with TBI and
Correspondence: Pascal Vachon Department of Veterinary Biomedicine, Faculty of Veterinary Medicine, University of Montréal, 3200 Sicotte, St-Hyacinthe, QC J2S 7C6, Canada

Tel: + I 450773852 I ext 8294

Email pascal.vachon@umontreal.ca 
the risk of central pain sensitization, chronic headaches, and low recovery rate if the pain is not treated..$^{5-7}$ In human patients, a precautionary principle prevails regarding analgesic medication for TBI patients, as practitioners fear greater cognitive and motor deficits with analgesia in the setting of BBB breakdown. However, this precautionary pain intervention protocol is based only on clinical experience. ${ }^{2}$ On the other hand, veterinarians are less prone to avoid analgesics to relieve their TBI patients, possibly because cognitive functions and headaches are more difficult to evaluate in animals. ${ }^{8}$ Also, the owners are less demanding regarding memory and fine motor control of their pets. In both cases, there is no evidence to support the benefit, or lack thereof, of analgesics or sedatives for neurologic care of TBI. ${ }^{9}$ Furthermore, there are only scarce data available pertaining to the choice of a specific analgesic for the treatment of TBI, as well as appropriate pain treatment protocols. ${ }^{10}$ If it could be shown that certain analgesics are not deleterious to the brain in a TBI situation, great benefits could be provided for patients. However, if the opposite is demonstrated, this would confirm current practice in human medicine and influence the veterinarian's intervention protocol for TBI.

The current study aimed to assess pain and motor behaviors following different doses of fentanyl in a rat model of IH and to evaluate the associated histopathology. Fentanyl was chosen for this study, because as with morphine, it is the most widely used analgesic to relieve pain associated with TBI. ${ }^{11}$ Also, fentanyl is very efficient to relieve intense pain, and, unlike morphine, it does not provoke histamine release, contributing to hemodynamic effects that may not be desirable in this pathology. ${ }^{12}$ Furthermore, it acts rapidly, has a shorter half-life than most opioids, and can be reversed by a $\mu$-receptor antagonist (ie, naloxone), making it safer to use than other analgesics. ${ }^{12,13}$ The recommended subcutaneous (SC) dose of fentanyl for rats is between 40 and $160 \mu \mathrm{g} / \mathrm{kg}$. With this posology, the analgesic effects are seen for 2 h. ${ }^{14,15}$ Fentanyl was selected since it was shown to be the most effective pain-relieving drug in rats. ${ }^{14}$ The optimal goal would be to maximize fentanyl use to obtain a better treatment protocol for TBI patients, which would relieve pain without causing any motor or cognitive deficits. This study did not address possible cognitive changes with analgesic treatment.

\section{Materials and methods}

\section{Animals}

Twenty-four specific pathogen-free male Sprague Dawley rats (Charles River, St-Constant, QC, Canada), weighing between
300 and $380 \mathrm{~g}$, at the beginning of the study, were used for this study. Animals were randomly assigned to experimental groups. They were kept in a standard laboratory animal environment (filtered air: 15 changes $/ \mathrm{h}$, humidity: 40\%-60\%, temperature: $21^{\circ} \mathrm{C} \pm 3{ }^{\circ} \mathrm{C}$, and light-dark cycle: $12: 12 \mathrm{~h}$ ). Rats were pair-housed in polycarbonate cages (Ancare, Bellmore, NY, USA) with hardwood bedding (Beta chip; Northeastern Products Co, Warrensburg, NY, USA). Animals were acclimated for 7 days prior to the beginning of the study. A polyvinyl chloride (PVC) tube was placed in each cage for environmental enrichment. Rats were given tap water and laboratory rat diet ad libitum (Charles River Rodent Chow 5075). The experimental protocol was approved by the Institutional Animal Care and Use Committee prior to animal use, and it is compliant with the Canadian Council on Animal Care guidelines (1993).

\section{Surgical procedures and collagenase injection}

To induce a standardized $\mathrm{IH}$, animals were anesthetized with vaporized isoflurane (3\% induction, $2 \%$ maintenance; Aerrane; Baxter, Mississauga, ON, Canada) in oxygen $(0.5 \mathrm{~L} / \mathrm{min})$. After hair clipping and disinfection of the cranial skin with proviodine, rats were placed in a stereotaxic apparatus (David Kopf Instruments, Tujunga, CA, USA) in aseptic conditions, before undergoing surgery. A $2 \mathrm{~cm}$ sagittal incision was made on the skin and periosteal membrane. After exposing the cranial bone, a burr hole measuring $1.5 \mathrm{~mm}$ in diameter was drilled at stereotaxic coordinates with reference to bregma (anteroposterior $0.0 \mathrm{~mm}$ and lateral $3.0 \mathrm{~mm}$ ) to attain the caudoputamen nucleus. Using a $5 \mu \mathrm{L}$ Hamilton syringe, $2 \mu \mathrm{L}$ of a collagenase solution consisting of $0.4 \mathrm{U}$ Collagenase Type IV (powder in saline; Life Technologies, Carlsbad, CA, USA) was then injected over a period of $10 \mathrm{~min}$ through the burr hole in the right caudoputamen nucleus, $5.0 \mathrm{~mm}$ below the dura. The needle was left in place for an additional 5 min to prevent backflow. It was then removed and the skin was sutured with five simple interrupted stitches (silk 2-0). Throughout the surgery, rats were on a heating pad; their rectal temperature was monitored (Thermalert TH-8; Physitemp, Clifton, NJ, USA) and maintained within $35.5^{\circ} \mathrm{C}-36.5^{\circ} \mathrm{C}$.

\section{Treatments}

The collagenase-injected animals received an SC injection $2 \mathrm{~h}$ following surgery and on the morning of the 2 following days. Rats in the control group $(n=6)$ received saline only, in a similar volume $(0.2 \mathrm{~mL})$ to the fentanyl high dose treated 
animals. Animals in each experimental group received 5 $(\mathrm{n}=6), 10(\mathrm{n}=6)$, or $20(\mathrm{n}=3) \mu \mathrm{g} / \mathrm{kg}$ of fentanyl (Fentanyl citrate, $50 \mu \mathrm{g} / \mathrm{mL}$; Sandoz, Boucherville, QC, Canada). Fentanyl doses were less than the recommended doses (40-160 $\mu \mathrm{g} / \mathrm{kg})^{14,15}$ considering the breakdown of the BBB in this animal model. Only 3 animals received the $20 \mu \mathrm{g} / \mathrm{kg}$ dose since they were clearly overdosed, showing signs of catalepsy and whole body rigidity for a period of 15-20 min following the fentanyl injection. Since animals recuperated well from this dose, it was then decided to continue the study with only 3 animals in this treatment group. The observer (LS) tasked with the rat evaluations was blinded to all treatments.

Three animals formed a sham group, where the same surgical procedures were performed as for the collagenaseinjected animals, except that saline was injected intracerebrally, and they were treated with $10 \mu \mathrm{g} / \mathrm{kg}$ of fentanyl SC for 3 consecutive days and evaluated on behavioral tests.

\section{Behavioral evaluations}

A week prior to the surgery, all rats were accustomed to the neurological and motor tests for 5 days. Experimental groups were then formed, and over the last 2 days, baseline scores were recorded. Figure 1 shows the study protocol timeline. The rat grimace scale (RGS), evaluated with videos, was used to evaluate pain behavior. Each rat was filmed prior to and following the SC injection on the day of the surgery (day 1) and on 2 subsequent days (days 2 and 3). Motor behavioral tests were then performed in the following order: neurological examination, the balance beam test, and the rotarod test. They were done on 5 subsequent days starting $24 \mathrm{~h}$ following the surgery (days 2-6). All behavioral tests were performed and evaluated by an observer (LS) blinded to the treatments.

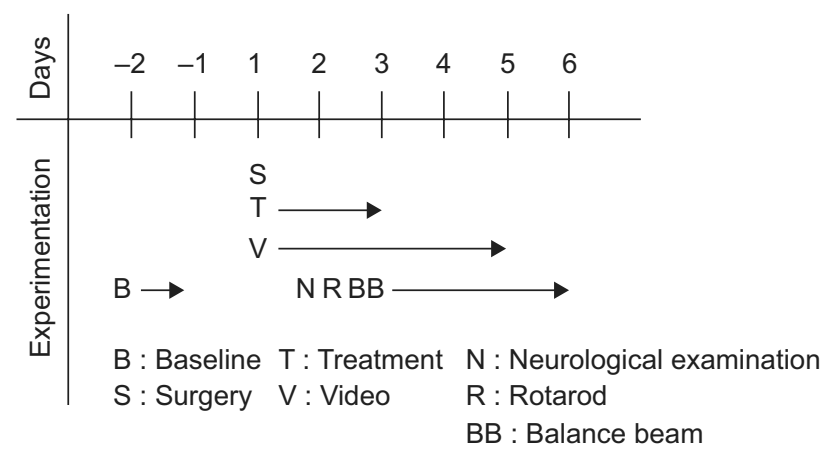

Figure I Study protocol timeline.

Notes: A week prior to the surgery, all rats were accustomed to the neurological and motor tests for 5 days and baseline scores were recorded on the last 2 days. Stereotaxic surgeries were performed (day I) and all animals were treated 2 hours following the surgery and on 2 subsequent days (days 2 and 3). Each rat was filmed before and after treatment and on days 4 and 5 . The neurological examination, the balance beam test, and the rotarod test were performed on days 2-6.

\section{RGS and pain evaluation}

In this study, rats were exposed to pain caused by the $\mathrm{IH}$ lesion. The brain parenchyma itself does not have pain receptors, but the nervous fibers surrounding the blood vessels and the afferent pathways will be sensitive to the neuroinflammation, high intracranial pressure, and ischemia. ${ }^{8}$ To evaluate this pain, rats were filmed for a 2 min duration sequence for each take (Logitech Webcam C210; Logitech, Newark, CA, USA) in a quiet, dimmed light room, before treatment and on 3 occasions, at 15 min intervals, starting $1 \mathrm{~h}$ after the treatment (days 1, 2, and 3). Videos were also taken on days 4 and 5 (days without treatment). Videos were analyzed following the Sotocinal's RGS method, described in detail in their manual. ${ }^{16}$ This scoring has shown very good interobserver and intraobserver reliability (with 0.85 [0.78-0.90, $95 \%$ confidence interval] and 0.83 [0.76-0.89], respectively) in other studies. ${ }^{17}$ The mean of the data collected from the 3 videos following treatments was used for analysis. Scoring included four action units: orbital tightening (narrowing of the orbital area, displaying a partial or complete eye closure or eye "squeezing"), nose/cheek flattening (display of less bulging of the nose and cheek, with possibly the absence of the crease between the cheek and whisker pads), ear changes (folded, curled, and angled forward or outward, resulting in a pointed shape; the space between the ears can look wider), and whisker changes (pointed forward, far from the face and may bunch up). Each of the action units was given an intensity rating ( 0 : absent; 1 : moderate or equivocation over its presence or absence; 2 : pronounced action units); then an RGS score was calculated by averaging all intensity ratings for each video. ${ }^{16}$ The maximal score reported is the mean of the action units (maximal value $=2$ ). All videos were evaluated on the entire sequence of each take by an observer (LS) who was blind to the treatment groups.

\section{Neurological examination}

The neurological examination included semiquantitative tests from previous published studies ${ }^{18-21}$ from the lab. Animals were evaluated following the surgery for 5 consecutive days starting $24 \mathrm{~h}$ after the surgery. The value reported is the sum of all tests (maximum score of 24) which consisted of the following:

- Activity: exploration of the immediate environment when the rat is placed on a novel hard surface ( 0 : no exploration; 1: exploration with head movements only; 2 : exploration of the immediate environment; 3: normal exploration);

- Locomotion: the rat is placed on a hard surface and forward progression is observed ( 0 : no displacement; 1 : 
unilateral rotation only; 2 : incomplete body movements on both sides, forward progression mainly by rotation; 3: normal linear progression);

- Visual positioning: the rat is held by the tail above a contact surface, then moved toward a hard surface and the visual positioning of both limbs is evaluated (1: contact with one limb only, other limb held in a flexed position; 2 : partial flexion of the affected limb; 3: both limbs extended to make contact and normal walking movements);

- Tremor: animal is held by the tail in an elevated position so that the front paws remain in contact with a hard surface; stability of hind paws is observed (1: tremor; 2 : no tremor);

- Pelvic limbs hypertonicity: animal is held by the tail in an elevated position so that the front paws remain in contact with a hard surface; rigidity of hind limbs is observed as a hyperextension of the hind legs (1: hypertonicity; 2 : normal tonicity);

- Tail rigidity: the tail is elevated at mid-length and rigidity is observed (1: no flexibility observed, if placed in a curved position remains as such; 2 : moderate rigidity; 3 : normal flexibility and movements);

- Climbing: climbing on a wire grid to observe skill and symmetry of forelimbs (1: holds wire but cannot let go; 2: tries to climb but has difficulty doing so, little forward movement with important asymmetry; 3: climbs but the movement is moderately asymmetrical; 4: normal climbing, symmetrical movements of both forearms are observed);

- Positional passivity: during hand restraint, the affected posterior limb (left) is pulled away from the animal and the motor response is observed ( 0 : no flexion of the limb; 1: flexion movements on occasions; 2 : normal flexion upon each extension);

- Hopping: the rat is held by its hind legs and one forepaw so that the entire weight of the animal is supported by one limb. The animal is moved laterally and hopping movements are evaluated to assess postural adjustments ( 0 : no postural adjustment; 1 : delay of the initiation of movement to adjust posture of the affected limb [left]; 2 : normal postural adjustment).

\section{Balance beam test}

A custom-made balance beam (wooden rod $80 \mathrm{~cm}$ in length and $2.8 \mathrm{~cm}$ in diameter) was placed over an open table (20 cm distance) with a PVC pipe (10 cm diameter) at the end (as an incentive to move forward) to evaluate motor coordination and balance of the rat crossing the beam without falling. The fastest time of a maximal of 3 consecutive trials was recorded.
If the rat was unable to move forward, its ability to stay balanced on the beam was observed. This test was performed following the neurological examination.

\section{Rotarod treadmill}

A rotarod treadmill (ENV576; Med Associates, St Albans, VT, USA) was used to test the motor coordination of the rats prior to surgery and in the recuperation postsurgical phase. The rotarod was set to accelerate over a 5 min period from 2.5 to $25 \mathrm{rpm}$; the maximal time performance of each rat was recorded (maximum: $5 \mathrm{~min}$ ). The value reported for each animal is the percentage of the baseline values. This test was performed following the balance beam test.

\section{Perfusion, histological procedures, immunohistochemistry, and image analysis} After the last neurological examination (day 6), rats were anesthetized with an intraperitoneal injection of $200 \mathrm{mg} / \mathrm{kg}$ of ketamine (Ketalean; Bimeda-MTC, Cambridge, ON, Canada) and $5 \mathrm{mg} / \mathrm{kg}$ of xylazine (Xylamax; Bimeda-MTC) and then perfused using the following method. The abdominal skin and muscles were cut and a pair of forceps was used to clamp the descending aorta. The thoracic cavity was then exposed, the right atrium was cut and the rats were perfused through the heart (right ventricle) with a physiological dextrose-sucrose solution $(100 \mathrm{~mL}$ per rat; solution composition for $1 \mathrm{~L}: 8 \mathrm{~g}$ $\mathrm{NaCl}, 4 \mathrm{~g}$ dextrose, $8 \mathrm{~g}$ sucrose, $0.23 \mathrm{~g}$ calcium chloride). ${ }^{19-21}$ All perfusion products were purchased from Sigma Inc ( $\mathrm{St}$ Louis, MO, USA). A second perfusion immediately followed using a $10 \%$ neutral-buffered formalin solution (100 $\mathrm{mL}$ per rat). Brains were removed in whole and fixed in the formalin solution for $48 \mathrm{~h}$ and then embedded in paraffin for slide preparation. Sections of $5 \mu \mathrm{m}$ thickness (one every $200 \mu \mathrm{m}$ from the beginning of the hematoma) were stained with hematoxylin, eosin, phloxine, and saffron (HEPS). Each brain section was photomicrographed using an AxioImager M1 microscope (Carl Zeiss Microscopy GmBH, Oberkochen, Germany) and the area occupied by the hematoma was determined by using Zen 2012 blue edition software (Carl Zeiss Microscopy $\mathrm{GmBH}$ ). Then the hematoma volume was estimated by multiplying the hematoma area of each section by the distances separating two consecutive sections (200 $\mu \mathrm{m}$ ). Three sections (adjacent to the second, third, and fourth HEPS-stained section) were stained with Fluoro-Jade $\AA$ $\mathrm{B}$, and the number of necrotic neurons was counted in the penumbral area of the hematoma (average of four areas [top, bottom, right, left] at 10X). Only cells that showed typical green fluorescence (excitation peak at $480 \mathrm{~nm}$ and 
emission peak at $525 \mathrm{~nm}$ ) were counted. Two sections (adjacent to the second and fourth HEPS-stained section) were processed with immunohistochemistry for glial fibrillary acidic protein (GFAP) executed using polyclonal antibodies (immunoglobulin fraction of rabbit antisera diluted at 1:500 in phosphate-buffered saline [pH 7.6] with $1 \%$ bovine serum albumin; BioGenex Laboratories, San Ramon, CA, USA) to determine the number of reactive astrocytes. Astrocytes were counted in the penumbral area of the hematoma (average of four areas [top, bottom, right, left] at 20×); all astrocytes had well-developed processes and intense GFAP immunoreactivity, consistent with reactive astrocytes.

\section{Statistical analysis}

For all behavioral tests an analysis of variance (ANOVA) was performed on repeated measures with the period (before vs after) as the intrasubject factor and treatment $(5 \mu \mathrm{g} / \mathrm{kg}$, $10 \mu \mathrm{g} / \mathrm{kg}$, and saline). Cochran-Mantel-Haenszel test (semiquantitative data) for repeated measures was done to evaluate changes over time ( 5 days). For the rotarod results, a priori contrasts were performed adjusting the alpha of each comparison with the Benjamini-Hochberg sequential method (quantitative data). For the statistical analysis of histological findings, a one-way ANOVA with post hoc Tukey's tests was performed.

\section{Results}

\section{Grimace pain score}

Figure 2 shows the grimace pain score results. Treatments showed statistically significant differences on day 1 (F2,15=11.8; $P<0.001)$, day 2 (F2,15=15.5; $P<0.001)$, and day $3(\mathrm{~F} 2,15=5.2 ; P<0.02)$ only. The fentanyl group $5 \mu \mathrm{g} / \mathrm{kg}$ (Figure 2A) showed a significant difference with the control

B

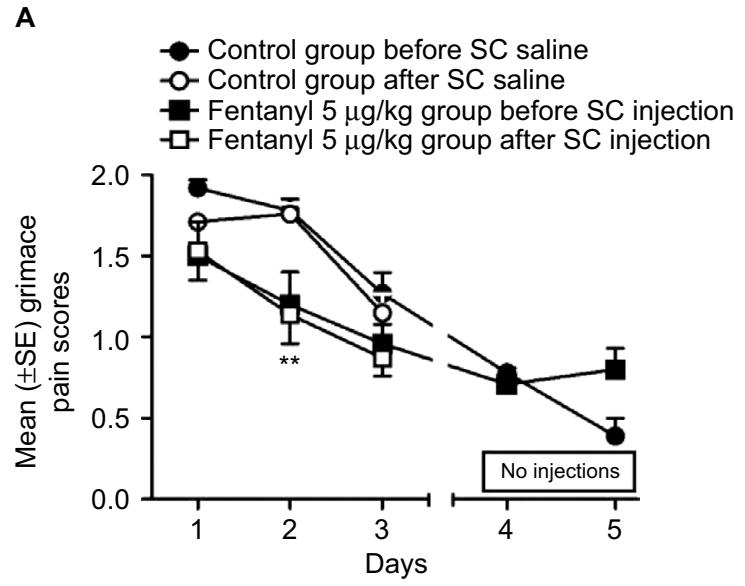
- Control group before SC saline
- Control group after SC saline
$\approx$ Fentanyl $10 \mu \mathrm{g} / \mathrm{kg}$ group before SC injection
$\rightarrow$ Fentanyl $10 \mu \mathrm{g} / \mathrm{kg}$ group after SC injection

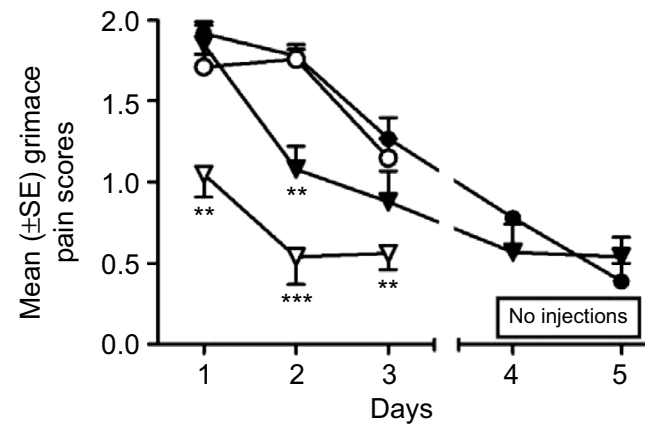

C $\quad \rightarrow$ Control group before SC saline -o-Control group after SC saline - Fentanyl $20 \mu \mathrm{g} / \mathrm{kg}$ group before SC injection $-\triangle$ Fentanyl $20 \mu \mathrm{g} / \mathrm{kg}$ group after SC injection

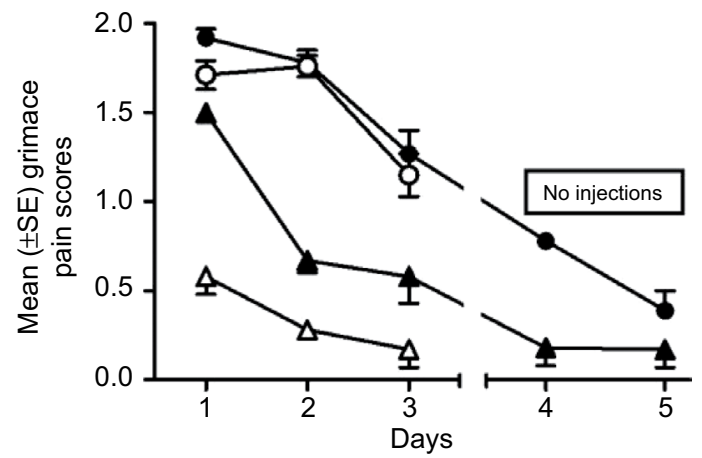

Figure 2 Grimace pain scores for saline and fentanyl treatment groups.

Notes: The surgery was performed on day I. The fentanyl $5 \mu \mathrm{g} / \mathrm{kg}$ treatment group (A) showed a significant difference with controls only on day 2 before and after the SC injection. The $10 \mu \mathrm{g} / \mathrm{kg}$ fentanyl treatment group (B) showed a significant difference with controls after injection on day I, and before and after injection on day 2 . On day 3 , only post-fentanyl administrations alleviated pain. No differences were noted on days 4 and 5 , on which animals were not treated. Due to the small number of subjects ( $\mathrm{n}=3$ ), no statistic was performed for the $20 \mu \mathrm{g} / \mathrm{kg}$ fentanyl treatment group (C). Results for the $20 \mu \mathrm{g} / \mathrm{kg}$ treatments seem to follow the same tendency as the $10 \mu \mathrm{g} / \mathrm{kg}$ treated animals although the analgesic response appears much more important for these animals. $* * P<0.01$, $* * * P<0.001$.

Abbreviations: SC, subcutaneous; SE, standard error. 
group on day $2(P<0.01$; contrast $t$-test values [before and after fentanyl] $=3.04$ and -3.31$)$, before and after the injection on that day. The fentanyl group $10 \mu \mathrm{g} / \mathrm{kg}$ (Figure 2B) showed a significant difference with controls; the pain was alleviated after injection on day $1(P<0.01$; contrast $t$-test value $=-3.03)$, and before and after injections on day $2(P<0.01$ and $P<0.001$; contrast $t$-test values [before and after fentanyl] $=-3.71$ and -6.53). And on day 3, only post-fentanyl administrations relieved pain $(P<0.01$; contrast $t$-test value $=-3.23)$. No statistic was performed for the fentanyl group $20 \mu \mathrm{g} / \mathrm{kg}(\mathrm{n}=3)$ (Figure 2C); however, the pain score was well below that of the fentanyl group $5 \mu \mathrm{g} / \mathrm{kg}$, and this occurred everyday. Also, it indicates that the $20 \mu \mathrm{g} / \mathrm{kg}$ group seems to follow the same tendency as the $10 \mu \mathrm{g} / \mathrm{kg}$ group. On days 4 and 5 , no treatments were performed and treatment groups were not significantly different on the grimace pain score from saline controls. It is important to note that the pain score was much lower on those 2 last days for every group.

\section{Neurological examination and individual tests}

The results of the total score of the neurological examination are presented in Figure 3. All groups were severely affected following the surgery $(60 \%-65 \%$ of baseline scores on the day following the surgery) and recuperated gradually over time (up to $75 \%-80 \%$ of their baseline score on day 6 ). No statistically significant difference was seen between the groups $(\mathrm{F}(2,15)=0.32 ; P=\mathrm{ns})$. Figure $4 \mathrm{~A}$ represents the results of each test for the saline group; there is a statistically significant difference for the hopping score over time $(P<0.01$; mean score differences $=13.2$ ). Figure 4 also shows the hop-

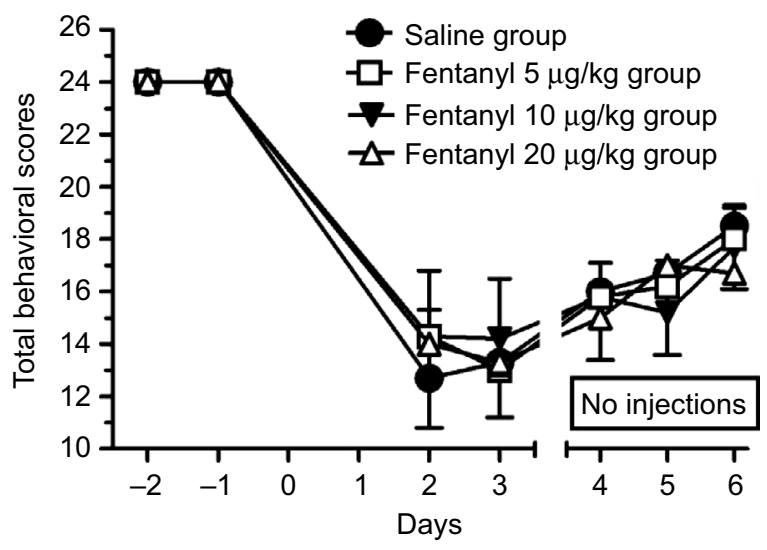

Figure 3 Neurological examination scores.

Notes: All groups were severely affected following the surgery $(60 \%-65 \%$ of baseline scores on the day following the surgery) and recuperated gradually over time (up to $75 \%-80 \%$ of their baseline score on day 6 ). No significant difference is seen between control and treatment groups. ping results for the 5 (Figure 4B) and $10 \mu \mathrm{g} / \mathrm{kg}$ (Figure 4C) groups, which did not recuperate over time. No significant difference was observed for the other individual neurological tests when comparing different treatments on each individual day and over time.

\section{Balance beam test}

Before surgery, all groups had comparable baseline results on the time to cross the beam (saline: $3.6 \pm 1.4$, and $3.5 \pm 0.8$, $3.8 \pm 0.6$, and $4.4 \pm 1.0 \mathrm{~s}$ for 5,10 , and $20 \mu \mathrm{g} / \mathrm{kg}$ fentanyl, respectively). Following surgeries, results were very comparable between the groups. On day 2, all animals fell once placed on the beam. On day 3,17\% of the saline treated animals and $50 \%-66 \%$ of the fentanyl treated animals were able to stay balanced on the beam. On day $4,66 \%$ of animals in each group were able to stay on the beam and all animals were able to balance themselves on the beam on days 5 and 6 . None of the animals were able to move forward.

\section{Rotarod treadmill test}

Rotarod results are presented in Figure 5. All groups were severely affected following the surgery $(35 \%-50 \%$ of baseline scores on the day following the surgery) and recuperated gradually over time. Treatments showed statistically significant differences $(\mathrm{F}(2,15)=2.2 ; P<0.05)$. Only the 10 $\mu \mathrm{g} / \mathrm{kg}$ fentanyl treatment showed significant a priori differences on day $5(P<0.05$; contrast $t$-test value $=-4.41)$ and day $6(P<0.02$; contrast $t$-test value $=-6.44)$, with animals performing more poorly on these days than the control and the $5 \mu \mathrm{g} / \mathrm{kg}$ groups. The $20 \mu \mathrm{g} / \mathrm{kg}$ fentanyl group cannot be statistically significant because of the low number of subjects in this group, but as an indicator, they follow the same trend as the $10 \mu \mathrm{g} / \mathrm{kg}$ group. The 3 animals that were sham operated showed no difference on the rotarod treadmill following $10 \mu \mathrm{g} / \mathrm{kg} \mathrm{SC}$ of fentanyl for 3 consecutive days (days 1, 2, and 3 postoperatively [compared to baseline] $=99 \%, 101 \%$, and $103 \%$, respectively). These animals were moving freely in their cage 15-20 min following the surgery and showed no signs of pain during the grimace pain score evaluation. Their balance beam test showed no significant differences from baseline. And their neurological examination was also normal confirming previously published findings. ${ }^{19-21}$

\section{Histopathological results}

No statistically significant differences were found between the saline and fentanyl treated animals for the hematoma volume, and the number of necrotic neurons and reactive astrocytes. Mean values are nearly the same for all groups. 

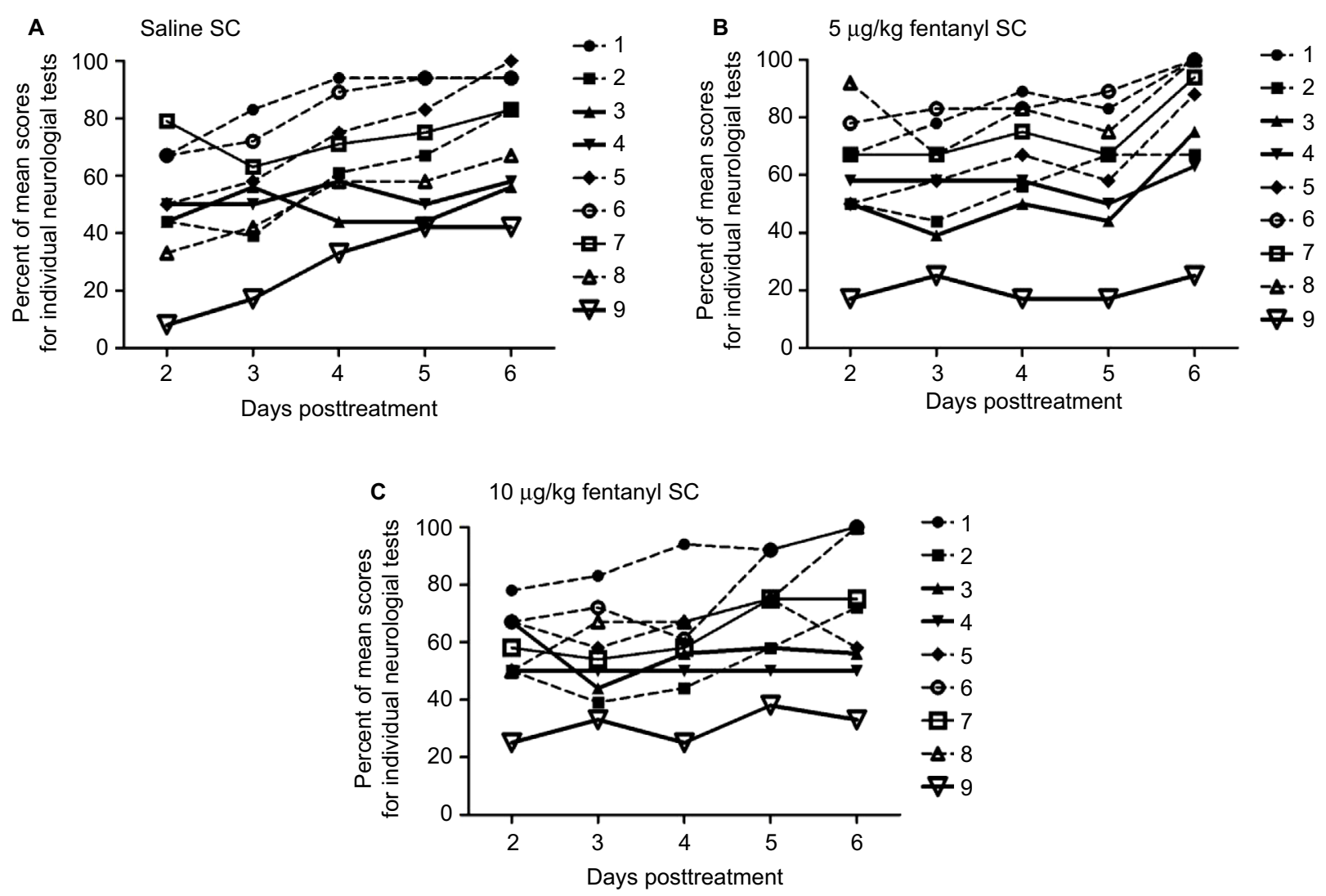

Figure 4 Results for individual neurological tests.

Notes: There is a statistically significant difference for the hopping score as function of time for the saline $(\mathbf{A})$ treated animals $(P<0.0 \mathrm{I})$. There is no significant difference in function over time for the 5 days (no amelioration with time) for the hopping test following the 5 (B) and $10 \mu g / \mathrm{kg}$ (C) fentanyl treatments. I: activity, 2: locomotion, 3: visual positioning, 4: tremor, 5: pelvic limbs hypertonicity, 6: tail rigidity, 7: climbing, 8: positional passivity, 9: hopping.

Abbreviation: SC, subcutaneous.

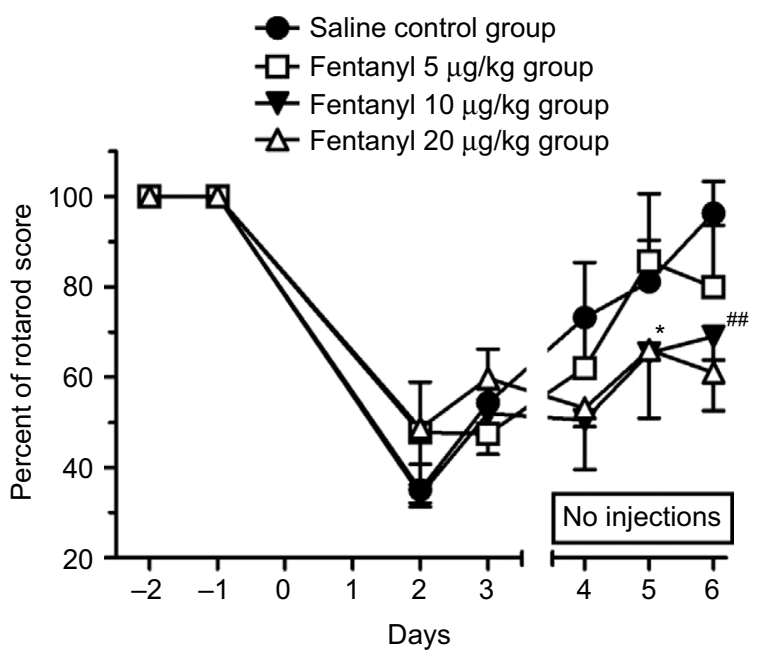

Figure 5 Rotarod treadmill test.

Notes: All groups were severely affected following the surgery $(35 \%-50 \%$ of baseline scores on day 2) and recuperated gradually over time. Only the $10 \mu \mathrm{g} /$ $\mathrm{kg}$ fentanyl treatment showed significant a priori differences on days 5 and 6, with animals performing more poorly on these days. No statistic was performed for the $20 \mu \mathrm{g} / \mathrm{kg}$ fentanyl group $(n=3)$; however, they seem to follow the same trend as the $10 \mu \mathrm{g} / \mathrm{kg}$ treatment group. $* P<0.05,{ }^{\# P} P<0.02$.
The mean hematoma volume ( \pm standard deviation $[\mathrm{SD}]$ ) for the saline, 5,10 , and $20 \mu \mathrm{g}$ fentanyl groups are, respectively, $11.9 \pm 2.33,12.1 \pm 1.0$, and $12.1 \pm 3.19 \mathrm{~mm}^{3}$. The mean total number of astrocytes $( \pm \mathrm{SD})$ for the saline, 5,10 , and 20 $\mu \mathrm{g}$ fentanyl groups are, respectively, $327 \pm 35.7,299 \pm 156.2$, and $284 \pm 70.0$. The mean total number of necrotic neurons $( \pm \mathrm{SD})$ for the saline, 5,10 , and $20 \mu \mathrm{g}$ fentanyl groups are, respectively, $16 \pm 6.3,16 \pm 8.3$, and $12 \pm 5.3$. The mean hematoma volume, total number of astrocytes, and total number of necrotic neurons for the $20 \mu \mathrm{g}$ fentanyl groups are, respectively, $12.6 \pm 3.87,304 \pm 137.1$, and $21 \pm 9.7 \mathrm{~mm}^{3}$.

\section{Body weights}

Only the saline control group gained weight (7\%) during the week following the surgery. All the fentanyl groups did not show a significant weight gain or loss during that period.

\section{Discussion}

The study shows that $10 \mu \mathrm{g} / \mathrm{kg}$ of fentanyl given SC can provide substantial analgesia in a collagenase-induced $\mathrm{IH}$ in 
rats. Even if fentanyl is considered a short action drug, its effects can last between 90 and $120 \mathrm{~min}$ if injected SC in a healthy rat. ${ }^{15}$ Furthermore, it has been suggested that conditions involving inflammation can affect the pharmacokinetics of drugs by affecting drug metabolizing enzymes and their transporters. The clinical results are hard to predict, but can include altered concentrations of the drug systemically or locally at the site of action. ${ }^{22}$ Also, inflammation in the brain, as seen in $\mathrm{IH}$ in animals and humans, is proven to disrupt the BBB allowing the migration of macromolecules from blood to the neuroparenchyma. ${ }^{23,24}$ This increased BBB permeability provides the opportunity for drugs like opioids to pass directly into the brain and accumulate, making them more effective. ${ }^{25,26}$ It is thus difficult to know the exact analgesic duration of fentanyl in these conditions. Group $10 \mu \mathrm{g} / \mathrm{kg}$ showed significantly less signs of pain during all 3 days of treatments and even before its treatment on day 2, implying that fentanyl offered substantial pain relief for a long period and prevented sensitization of the central nervous system (CNS) without changing motor behaviors.

For the motricity assessment of this study, the rotarod results clearly show a progressive improvement curve for all groups after surgery, except for the $10 \mu \mathrm{g} / \mathrm{kg}$ group on days 5 and 6 where they performed significantly below the results of saline and $5 \mu \mathrm{g} / \mathrm{kg}$ groups. This finding is also supported by the $20 \mu \mathrm{g} / \mathrm{kg}$ data that lean in the same direction. Sham group results suggest that the motricity was not affected by the fentanyl $(10 \mu \mathrm{g} / \mathrm{kg})$ injection alone, implying that the deficits seen in the IH rats were caused by IH combined with fentanyl, and not solely the dazing effect of the drug. Morphine has been shown to inhibit local production of cytokines during inflammation, and with chronic treatment to also decrease neutrophil and macrophage recruitment at the injury site. ${ }^{27,28}$ These findings suggest that fentanyl could reduce the damage caused by the local brain inflammation. Since the immunohistochemistry and histopathological results from the present study demonstrated no significant difference between the treatment groups, it is possible to conclude that fentanyl neither improves nor worsens brain lesions in IH. Regarding lesions in other brain areas, even though rupture of the BBB likely increased the amount of fentanyl in the brain tissue, this increase was probably limited to the penumbra, and the doses used are not known to be neurotoxic. Furthermore, the ipsilateral cortex and contralateral neuroparenchyma did not show any evidence of neurotoxicity in histopathology (HEPS), Fluoro-Jade staining, or GFAP immunohistochemistry.

Similarly, a study of Statler et al on the effects of different anesthetics in $\mathrm{TBI}^{29}$ showed that the worst results for motor and cognitive skills were associated with fentanyl and morphine. These drugs had no neuroprotective effect, and it was suggested that they failed to reduce the inflammation injury cascade caused by cerebral trauma. As in this experiment, the volume of the lesion was not affected by different analgesic treatments. Therefore, the decreased performance on the last 2 days for $10 \mu \mathrm{g} / \mathrm{kg}$ group could be explained by a functional interference of the drug. Morphine, another opioid agonist, has been shown to hinder the restructuration of new brain synapses. ${ }^{30}$ Cognitive deficits could be caused by a change of the gamma-aminobutyric acid-ergic (GABAergic) inhibition that controls neuronal excitation. Also, a loss of GABA-containing neurons is observed in the dentate gyrus of the hippocampus in morphine-exposed rats, thereby affecting the synaptic plasticity and spatial memory of the subjects. ${ }^{30}$ However, there are presently no known publications on the effects of fentanyl on neural plasticity. It is important to note that even with decreased motor capabilities, the $10 \mu \mathrm{g} /$ $\mathrm{kg}$ group was still recuperating. It is possible that with time they could catch up with the other groups.

The total score of the neurological examination does not show any difference between the groups as they all start at $60 \%-65 \%$ of the baseline score and recuperate around $75 \%-80 \%$ on day 6 , exhibiting a general improvement of their functions. However, taken individually, the hopping test stands out. It is always weakest on the first day, but there is a significant improvement of the score for the control saline group that fails to be present on the scores of the two fentanyl groups. This tendency follows the lack of amelioration on the rotarod for the 10 and $20 \mu \mathrm{g} / \mathrm{kg}$ groups, indicating that the use of fentanyl with IH will more strongly affect the spatial proprioception of the subjects since it is involved in both tasks.

The balance beam did not deliver as much information as was hoped. This test was supposed to evaluate walk progression on the beam, but instead it only showed if rats would fall off or could balance themselves on the beam. The only interesting result was that on day 2 fentanyl groups were quicker to be able to stand on the beam compared to the saline group. This observation could probably be explained by the fact that fentanyl subjects were in less pain on day 2 , given that starting on day 3 all groups did the same performance for the rest of the experimentation. It is concluded that this test is too demanding for the $\mathrm{IH}$ rat model and it does not provide interesting data for this kind of study.

The $20 \mu \mathrm{g} / \mathrm{kg}$ group was downsized to only 3 rats, after noticing signs of catalepsy and body rigidity following the fentanyl injection. Those signs were observed for the first 15-20 min posttreatment; thus, when the videos were taken an hour postinjection the rats had returned to normal. Those symptoms are clearly an indication of opioid overdose, 
despite the suggested SC fentanyl analgesic dose for rats ranging from 40 to $160 \mu \mathrm{g} / \mathrm{kg} .{ }^{15}$ This phenomenon suggests what clinicians already knew in that drugs affecting the CNS are more potent with BBB breakdown and their usual dose should be reduced with care. ${ }^{31}$

\section{Limitations}

The use of only one drug, fentanyl, is one of the limitations of this study. It would be interesting to perform further research with different analgesics that would provide better motor and cognitive recuperation. Ketamine, an N-Methyl-D-aspartate antagonist with analgesic properties, could be an option since it has anti-excitotoxic and anti-apoptotic properties..$^{32}$ However, according to Statler et al, ${ }^{29}$ it would be a poor choice as it had the worst hippocampal neuronal survival and may exacerbate hypoperfusion and/or hippocampal glucose utilization. Diazepam could be a better alternative since it only shows a trend toward longer latency on the motor tests..$^{29}$ Also, it would be interesting to test the motor and cognitive effect of gabapentin in a TBI model, as it is used as an antineuropathic pain medication and to prevent central pain sensitization; $;^{33,34}$ however, some authors have shown limited efficiency with this molecule. ${ }^{35}$

\section{Conclusion}

The use of $10 \mu \mathrm{g} / \mathrm{kg}$ of fentanyl administered SC to relieve pain in rats suffering from IH seems safe and efficient. However, the analgesic had an effect on some motor evaluations, notably the hopping test and the rotarod treadmill. Disregarding these effects could represent an important bias in future studies. In summary, a dose of $10 \mu \mathrm{g} / \mathrm{kg}$ of fentanyl is recommended to relieve the pain associated with a TBI rat model, while taking into account the possible deficits in experimentation planning.

\section{Acknowledgments}

This study was supported by the Fond de Développement pour la Médecine des Animaux de Laboratoire (Pascal Vachon). The authors would like to acknowledge the assistance of Guy Beauchamp (Faculty of Veterinary Medicine University of Montreal) for statistical analyses.

\section{Disclosure}

The authors report no conflicts of interest in this work.

\section{References}

1. Lu J, Marmarou A, Choi S, Maas A, Murray G, Steyerberg EW. Mortality from traumatic brain injury. Acta Neurochir Suppl. 2005;95:281-285.

2. Brain Trauma Foundation; American Association of Neurological Surgeons; Congress of Neurological Surgeons. Guidelines for the management of severe traumatic brain injury. J Neurotrauma. 2007;24(Suppl 1) S1-S106.
3. Morgenstern LB, Hemphill JC 3rd, Anderson C, et al. Guidelines for the management of spontaneous intracerebral hemorrhage: a guideline for healthcare professionals from the American Heart Association/ American Stroke Association. Stroke. 2010;4:2108-2129.

4. Jauch EC, Saver JL, Adams HP Jr, et al. Guidelines for the early management of patients with acute ischemic stroke: a guideline for healthcare professionals from the American Heart Association/American Stroke Association. Stroke. 2013;44:870-947.

5. Burstein R, Goor-Aryeh I, Jakubowski M. Sensitization of trigeminovascular neurons and migraine therapy with triptans. In: Villanueva L, Dickenson AH, Ollat H, editors. The Pain System in Normal and Pathological States: A Primer for Clinicians. Progress in Pain Research and Management. Seattle: IASP Press; 2004:211-222.

6. Grant D. Pain Management in Small Animals. New York: ButterworthHeinemann/Elsevier; 2006.

7. Williams E. Nursing perspective on pain management. In: Vadivelu N, Urman RD, Hines RL, editors. Essentials of Pain Management. New York: Springer New York; 2011:367-377.

8. Gregory NG. Physiology and Behaviour of Animal Suffering. Oxford, UK; Ames (IW): Blackwell Science; 2004.

9. Teitelbaum JS, Ayoub O, Skrobik Y. A critical appraisal of sedation, analgesia and delirium in neurocritical care. Can J Neurol Sci. 2011;38(6):815-825.

10. Torbey MT, Bosel J, Rhoney DH, et al. Evidence-based guidelines for the management of large hemispheric infarction: a statement for health care professionals from the Neurocritical Care Society and the German Society for Neuro-Intensive Care and Emergency Medicine. Neurocrit Care. 2015;22(1):146-164.

11. Shapiro BA, Warren J, Egol AB, et al. Practice parameters for intravenous analgesia and sedation for adult patients in the intensive care unit: an executive summary. Society of Critical Care Medicine. Crit Care Med. 1995;23(9):1596-1600.

12. Chauvin M, Beaulieu P. Pharmacologie des opioïdes. In: Beaulieu P, editor. Pharmacologie de la douleur. Montréal (QC): Les Presses de l'Université de Montréal; 2005:39-77.

13. Deschamps J-Y. Vade-mecum de gestion de la douleur chez le chien et le chat. Gestion de la douleur chez le chien et le chat. Paris: Med'com; 2010.

14. Meert TF, Vermeirsch HA. A preclinical comparison between different opioids: antinociceptive versus adverse effects. Pharmacol Biochem Behav. 2005;80(2):309-326.

15. van den Hoogen RH, Colpaert FC. Epidural and subcutaneous morphine, meperidine (pethidine), fentanyl and sufentanil in the rat: analgesia and other in vivo pharmacologic effects. Anesthesiology. 1987;66(2):186-194.

16. Sotocinal SG, Sorge RE, Zaloum A, et al. The Rat Grimace Scale: a partially automated method for quantifying pain in the laboratory rat via facial expressions. Mol Pain. 2011;7:55.

17. Oliver V, De Rantere D, Ritchie R, Chisholm J, Hecker KG, Pang DS. Psychometric assessment of the Rat Grimace Scale and development of an analgesic intervention score. PLoS One. 2014;9(5):e97882.

18. Vachon P, Ste-Marie L, Montgomery J. Predictive value of a battery of behavioural tests to determine the extent of induced cerebral ischemia by cauterisation of the middle cerebral artery. Sci Tech Anim Lab. 1999;24(3):171-177.

19. Lema PP, Girard C, Vachon P. Evaluation of dexamethasone for the treatment of intracerebral hemorrhage using a collagenase-induced intracerebral hematoma model in rats. $J$ Vet Pharmacol Ther. 2004;27(5):321-328.

20. Lema PP, Girard C, Vachon P. High doses of methylprednisolone are required for the treatment of collagenase-induced intracerebral hemorrhage in rats. Can J Vet Res. 2005;69(4):253-259.

21. Savard C, Lema PP, Helie P, Vachon P. Effects of timing of dexamethasone treatment on the outcome of collagenase-induced intracerebral hematoma in rats. Comp Med. 2009;59(5):444-448.

22. Christensen H, Hermann M. Immunological response as a source to variability in drug metabolism and transport. Front Pharmacol. 2012;3:8. 
23. Keep RF, Xiang J, Ennis SR, et al. Blood-brain barrier function in intracerebral hemorrhage. Acta Neurochir Suppl. 2008;105:73-77.

24. Hawkins BT, Davis TP. The blood-brain barrier/neurovascular unit in health and disease. Pharmacol Rev. 2005;57(2):173-185.

25. Ronaldson PT, Davis TP. Targeting blood-brain barrier changes during inflammatory pain: an opportunity for optimizing CNS drug delivery. Ther Deliv. 2011;2(8):1015-1041.

26. Witt KA, Davis TP. CNS drug delivery: opioid peptides and the bloodbrain barrier. AAPS J. 2006;8(1):E76-E88.

27. Clark JD, Shi X, Li X, Qiao Y, Liang D, Angst MS et al. Morphine reduces local cytokine expression and neutrophil infiltration after incision. Mol Pain. 2007;3:28.

28. Martin JL, Koodie L, Krishnan AG, Charboneau R, Barke RA, Roy S. Chronic morphine administration delays wound healing by inhibiting immune cell recruitment to the wound site. Am J Pathol. 2010;176(2): 786-799.

29. Statler KD, Alexander H, Vagni V, et al. Comparison of seven anesthetic agents on outcome after experimental traumatic brain injury in adult, male rats. J Neurotrauma. 2006;23(1):97-108.
30. Niu L, Cao B, Zhu H, et al. Impaired in vivo synaptic plasticity in dentate gyrus and spatial memory in juvenile rats induced by prenatal morphine exposure. Hippocampus. 2009;19(7):649-657.

31. Armitage-Chan EA, Wetmore LA, Chan DL. Anesthetic management of the head trauma patient. J Vet Emerg Crit Care. 2007;17(1):5-14.

32. Engelhard K, Werner C, Eberspacher E, et al. The effect of the alpha 2-agonist dexmedetomidine and the N-methyl-D-aspartate antagonist $\mathrm{S}(+)$-ketamine on the expression of apoptosis-regulating proteins after incomplete cerebral ischemia and reperfusion in rats. Anesth Analg. 2003;96(2):524-531.

33. Attal N, Brasseur L, Parker F, Chauvin M, Bouhassira D. Effects of gabapentin on the different components of peripheral and central neuropathic pain syndromes: a pilot study. Eur Neurol. 1998;40(4):191-200.

34. Castel A, Vachon P. Gabapentin reverses central pain sensitization following a collagenase-induced intrathalamic hemorrhage in rats. $J$ Pain Res. 2013;7:5-12.

35. Yang F, Fu H, Lu YF, et al. Post-stroke pain hypersensitivity induced by experimental thalamic hemorrhage in rats is region-specific and demonstrates limited efficacy of gabapentin. Neurosci Bull. 2014;30(6):887-902.
Journal of Pain Research

\section{Publish your work in this journal}

The Journal of Pain Research is an international, peer reviewed, open access, online journal that welcomes laboratory and clinical findings in the fields of pain research and the prevention and management of pain. Original research, reviews, symposium reports, hypothesis formation and commentaries are all considered for publication.

\section{Dovepress}

The manuscript management system is completely online and includes a very quick and fair peer-review system, which is all easy to use. Visit http://www.dovepress.com/testimonials.php to read real quotes from published authors. 